\title{
Common Information Model Expansion for Distributed Wind Power
}

\author{
Yuehui HUANG ${ }^{1,}$, Dongying ZHANG ${ }^{2, b}$, Jiutong LIN ${ }^{2, c}$, Dewei LIU $^{1, d}$ \\ ${ }^{1}$ China Electric Power Research Institute, Beijing, 100085, China \\ ${ }^{2}$ North China Electric Power University, Beijing, 102206, China \\ aemail: huangyh@epri.sgcc.com.cn, bemail: zdyingmail@163.com, 'email: \\ jtlin1020@hotmail.com, demail: liudw@epri.sgcc.com.cn
}

\begin{abstract}
Keywords: Distributed Generation; Wind Power; Common Information Model; Class Extension; Energy Management System
\end{abstract}

\begin{abstract}
Large-scale distributed wind power connected to grid must be dispatched and managed by the provincial gird dispatch center. In order to unify representation of wind power equipment's information and function in various applications, this paper extends the common information model of the wind plant based on IEC61970 to meet the provincial dispatch automation system's data requirements of the grid-connected wind plant. Combined with the requirements of power system simulation, this paper also analyses the key factors that have an influence on the dynamic performance of wind turbines, categories its data requirements, and extends common dynamic information model of wind turbines. The proposed extended common information model of wind power fully satisfies the present requirements of data and function for the dispatch center's management of distributed wind power.
\end{abstract}

\section{Introduction}

To end the abandoned wind power phenomenon caused by centralized wind power and long distance transmission, decentralized wind power is proposed, which not only improves power quality and meets the power supply demand at the user end, but also adapts to the low-technological level status quo and effectively eases the supply pressure of highly loaded areas and remote areas. Currently China's wind power development is changing from centralized large-scale development to centralized large-scale development and distributed development.

New management problems has emerged with more and more decentralized wind power being connected to the grid. Generally, distributed wind power is connected to the grid which is managed by the local gird dispatch center. However, due to the limited power adjustment capacity of local grid dispatch center, it must be managed by provincial dispatch center to ensure power balance. Therefore, distributed wind power must be dispatched and managed by the provincial dispatch center. The devices which are dispatched need to establish the common information model (CIM) based on IEC61970 to ensure the consistency of information and functional expression in various applications [1-2]. The CIM extension principle is to use the existing CIM package as far as possible [3]. CIM extension mainly take three issues into consideration: whether to add new packages, what new classes to be added and which of the existing classes should be added with attribute values and attributes.

Current researches of CIM extension of wind power focus on simulation application in micro-grid and distributed network [4-9]. However, there is a certain gap between the research results and to the provincial dispatch center's actual demand for monitoring, forecasting, planning and simulation of DTS. This paper extends the CIM of wind plant and common dynamic information model of wind turbines based on the provincial dispatch automation system's data and functionality requirements.

\section{Wind Plant CIM Extension}

The production package describes various types of electric generators. This paper extends the 
wind plant model in the production package which meets the provincial dispatch automation system's data requirements of the grid-connected wind plant. It is as shown in Fig. 1.

The design classes WindPlant, WindTowerData, SVCData are different kinds of power system resource and inherit from class PowerSystemResource.

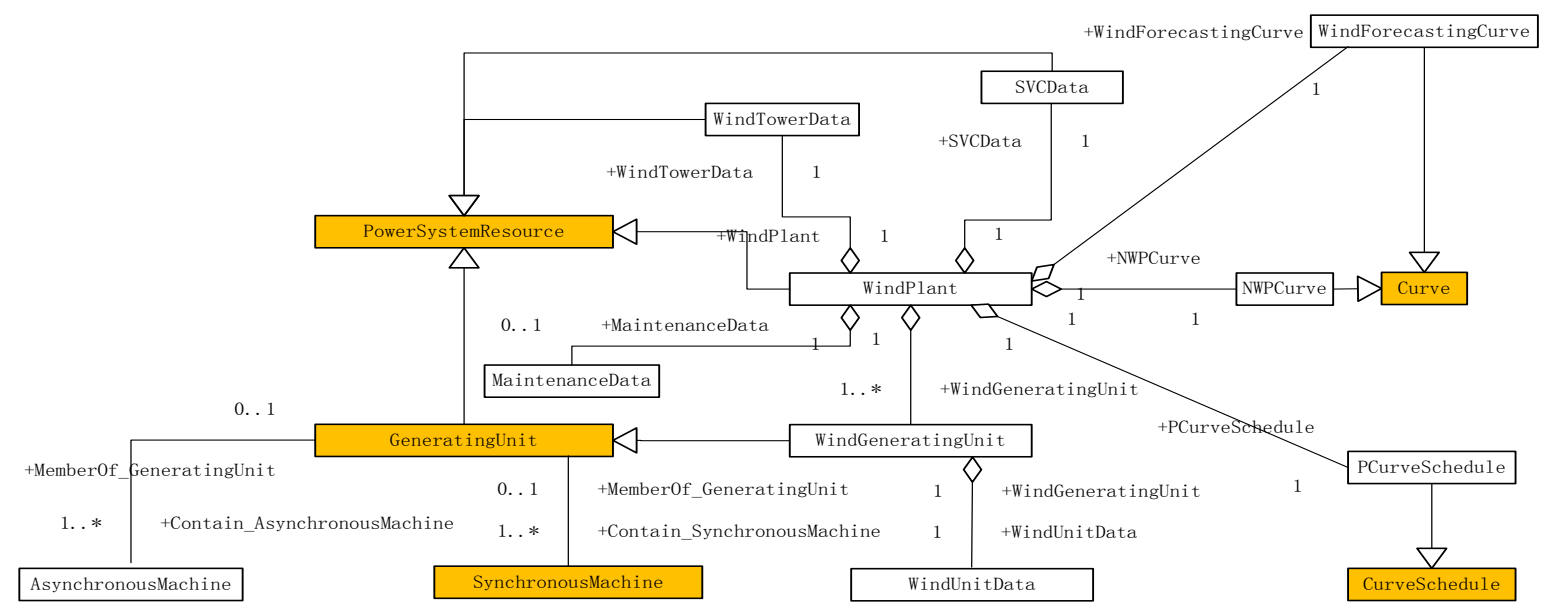

Fig. 1 Wind Plant Model

The design class WindGeneratingUnit forms a clustering relationship with class WindPlant.

Class WindGeneratingUnit inherits from class GeneratingUnit. Since wind generators include synchronous generator and asynchronous generator, class SynchronousMachine and AsynchronousMachine form an association relationship with class GeneratingUnit.

Since CIM contains a model for synchronous machine, a model for asynchronous machine therefore should be established. The AsynchronousMachine is designed with a reference to synchronous generator model. The design class AsynchronousMachine inherits from class RegulatingCondEg, associates with the class Terminal and thus forms a topological network with other conduction equipment.

Now the dispatch center at the provincial level requires wind plants to report forecasting, planning and real-time data. According to the need of these data, this paper designs new classes and their attributes, and illustrates their application and relationship with other classes.

(1) Short-term and ultra-short-term forecasting data

The content of short-term forecasting data is the wind plant output power, the estimated power capacity and the numerical weather prediction (NWP) which produces every fifteen minutes from 00:15 to 24:00 of the fourth day. This data should be reported to the provincial dispatch center once a day.

The content of ultra-short-term forecasting data is the wind plant output power, the real-time power capacity over the next four hours, which produces every fifteen minutes. The data of real-time power capacity is from the monitoring system of wind plant. The remote measurement of every unit adds up to the total capacity of the units. This data should be reported every fifteen minutes.

The design class WindForecastingCurve inherits from class Curve and forms a clustering relationship with class WindPlant. The design class WindForecastingCurve describes short-term output power and wind turbine power forecasting curve in the short-term forecast and can be applied to unit scheduling. The design class WindForecastingCurve describes ultra-short-term output power and real-time power capacity forecasting curve in the ultra-short-term forecast and can be applied to economic dispatch.

The design class NWPCurve inherits from class Curve and describes meteorological curve in NWP, which includes height, wind speed, wind direction, temperature, humidity, barometric pressure. It forms a clustering relationship with class WindPlant and can be applied to calibrate and revise the output power forecast. 
(2) Plan Curve of Active Power

The design class PCurveSchedule inherits from class CurveSchedule and describes planning curve of total active power of the wind plant in a short-term forecast. It form a clustering relationship with class WindPlant and can be applied to unit scheduling.

(3) Data of Maintenance Scheduling

The data is the start time and end time of maintenance, the name of maintenance equipment, and the number of units which are out of work caused by maintenance. The data should be reported every day.

The design class MaintenanceData, describing maintenance data, forms a clustering relationship with class WindPlant and can be applied to unit scheduling.

(4) Real-time Data of Wind Tower

The data is the number of wind tower, the average value of wind speed, wind direction, wind speed standard deviation, instantaneous wind speed, instantaneous wind direction, maximum wind speed, temperature, barometric pressure and humidity of all high levels in five minutes. The high levels include ten meters, thirty meters, fifty meters, sixty meters, seventy meters, eighty meters, ninety meters, and one hundred meters. The data should be reported every five minutes.

The design class WindTowerData, describing real data of wind tower, forms a clustering relationship with class WindPlant and can be applied to forecast and plan.

(5) Real-time Data of Wind Unit

The data includes number, model, longitude, latitude, active power, reactive power, head wind, wind direction, operation condition, A phase voltage of the wind unit. The operation condition includes Waiting, Operating, and Error. The data should be reported every five minutes.

The design class WindUnitData describes the real-time data of wind unit and forms a clustering relationship with class WindGeneratingUnit.

(6) Real-time Data of SVC

The data is number, capacity, and reactive power of SVC. The data needs to be reported every five minutes.

The design class SVCData describes the real-time data of SVC and forms a clustering relationship with class WindPlant.

\section{Wind Turbine Dynamic CIM Extension}

The GenerationDynamics package describes all kinds of prime movers and can be applied to simulation and training. The original GenerationDynamics package only has conventional prime movers, such as steam turbine CIM which describes the data model of the process of steam energy turning into mechanical energy. In order to simulate the dynamic process of wind energy turning into mechanical energy, class WindTurbine needs to be extended. The design class WindTurbine inherits from class PrimeMover.

According to the wind turbine structure, the dynamic model of wind turbine includes aerodynamic model, shaft model, pitch angle and speed control model. This article refines the basic data that can simulate the wind turbine's dynamic process, and its achieving approach, extends new classes and their attributes, and illustrates their relationship with other classes through sorting out principles of each model.

(1) Wind Turbine Aerodynamic Model

The wind turbine aerodynamic model is applied to calculate the mechanical power $P_{M}$ generated by wind wheel. $P_{M}$ can be obtained from calculating power coefficient curve. This curve is given by the manufacturer in form of $C_{p}(\lambda, \beta)$. $C_{p}$ is power factor. $\lambda$ is tip speed ratio. $\beta$ is pitch angle. $\lambda=\omega_{M} R / V$. $V$ is wind speed. $\omega_{M}$ is wind wheel speed. $R$ is radius of wind wheel.

The wind power in area of $\pi R^{2}$ is as follows:

$$
P_{V}=1 / 2 \rho_{A I R} V^{3} \pi R^{2}
$$

The mechanical power of wind wheel in any steady operating point is as follows: 


$$
P_{M}=P_{V} C_{P}(\lambda, \beta)
$$

In the steady-state case, $P_{M}$ minus losses is electromagnetic power $\left(P_{E}\right)$ of wind turbine.

In the case that wind units don't provide power coefficient curve, this article uses cut-in speed, rated speed, rated power, rated wind wheel speed and wind wheel radius to approximately calculate wind power factor and wind power curve.

So for a certain speed, $\omega_{M}$ and $\beta$ determine $P_{M}$.

(2) Pitch Angle and Speed Control Model

The wind unit with the pitch function has the pitch angle control model. The model can control pitch angle to make wind unit output power at a rated level when wind speed exceeds the rated speed. Curve $\beta(V)$ between pitch angle and speed is given by the manufacturer. It gathers generator rotor speed to reckon pitch angle in the actual control system. Compared with the transient change of grid, the response time of pitch servo system is much slower. The delay mechanism should be included in the model and the delay can be set as seconds. A maximum pitch rate should be set to reduce dynamic inflow during the pitch process.

It controls pitch angle at $\beta_{\text {ORDR }}$ when there is an external command of reducing power. $\beta_{\text {ORDR }}$ is pitch angle corresponding to the desired output power. $\beta_{O R D R}(V)$ corresponding to every speed is given by the manufacturer.

The wind unit with a shift function has the rotor speed control model. The model can control rotor speed and ensure that the wind unit outputs the maximum power when the wind speed is below the rated speed. Curve $\omega(V)$ between optimal rotor speed and wind speed is given by the manufacturer. It gathers generator rotor speed $\omega_{E}$ or electromagnetic power $P_{E}$ to reckon the corresponding electric power $P_{\text {ref }}$ based on curve between rotor speed and maximum power, and then adjust rotor speed through controlling electric power which is controlled by rotor excitation current.

The control systems provided by various manufacturers have a lot of differences and the internal data is hard to obtain. However, no matter how the control system is realized, its external characteristic reflects at $\beta(V), \beta_{\text {ORDR }}(V), \omega(V)$ which are provided by the manufacturer. These curves can be used as the basis in the dynamic simulation process of wind turbine.

(3) Shaft Model

In terms of the problem it studies, shaft model includes two-mass model and lumped-mass model and so on. Each mass model needs identifying inertia and stiffness parameter.

To sum up, it needs $C_{p}(\lambda, \beta), \beta(V), \beta_{\text {ORDR }}(V), \omega(V)$ and parameters of shaft model in the dynamic simulation process of wind turbine mechanical power.

The extended dynamic wind turbine model in the GenerationDynamics package is as shown in Fig. 2.

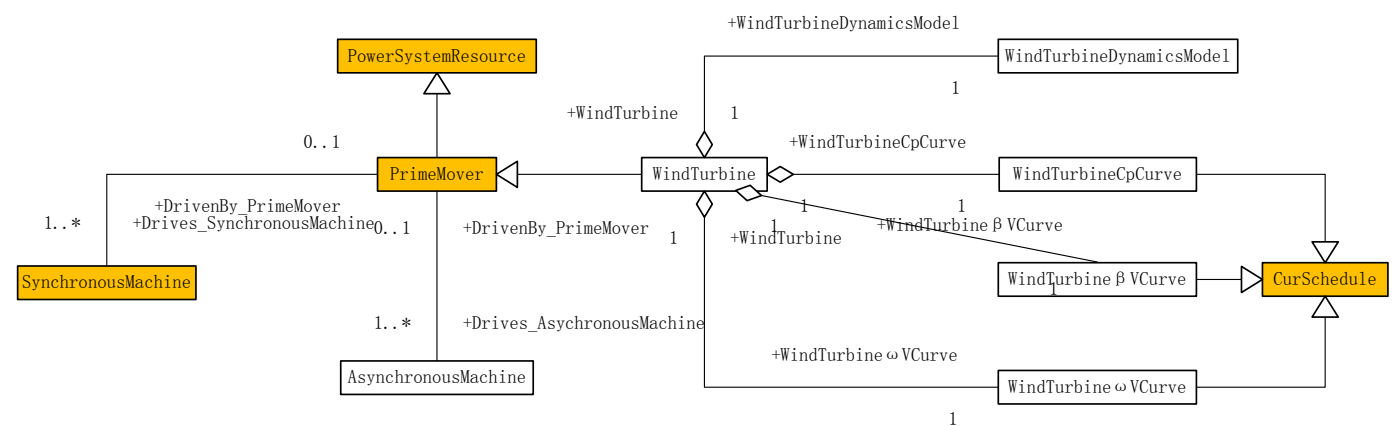

Fig. 2 WindTurbine Dynamic Model

The design classes, including WindTurbineCpCurve, WindTurbine $\beta$ VCurve and WindTurbine $\omega$ VCurve, inherit from class CurveSchedule, and form class WindTurbine combining class WindTurbineDynamicsModel describing shaft model.

Wind generator is driven by prime mover no matter whether it's a synchronous machine or a asynchronous machine. Thus, class SynchronousMachine and class AsynchronousMachine form an association relationship with class PrimeMover. 


\section{Summary of Extended Classed of Wind Generator}

Table 1 summarizes the extended classes of wind plant and the description of their attributes.

Table 1 Wind plant extended class

\begin{tabular}{|c|c|c|c|}
\hline New Class & Parent Class & Package & Description \\
\hline WindPlant & PowerSystemResource & Production & Wind Plant \\
\hline SVCData & PowerSystemResource & Production & Real Data of SVC \\
\hline WindTowerData & PowerSystemResource & Production & Real Data of Wind Tower \\
\hline WindGeneratingUnit & GeneratingUnit & Production & Wind Generator Unit \\
\hline WindTurbine & PrimeMover & $\begin{array}{l}\text { GenerationD } \\
\text { ynamics }\end{array}$ & Wind Turbine \\
\hline AsynchronousMachine & RegulatingCondEg & Wires & Asynchronous Machine \\
\hline ShortForecastingCurve & Curve & Production & Short Forecasting Curve \\
\hline NWPCurve & Curve & Production & NWP Curve \\
\hline $\begin{array}{l}\text { UltraShortForecastingCu } \\
\text { rve }\end{array}$ & Curve & Production & $\begin{array}{c}\text { Ultra Short Forecasting } \\
\text { Curve }\end{array}$ \\
\hline $\begin{array}{l}\text { PCurveSchedule } \\
\text { MaintenanceData } \\
\text { WindUnitData }\end{array}$ & CurveSchedule & $\begin{array}{l}\text { Production } \\
\text { Domain } \\
\text { Domain }\end{array}$ & $\begin{array}{l}\text { Plan Active Power Curve } \\
\text { Data of Maintenance } \\
\text { Real Data of Wind Unit }\end{array}$ \\
\hline WindTurbineCpCurve & Curve & $\begin{array}{l}\text { GenerationD } \\
\text { ynamics }\end{array}$ & Wind Turbine Cp Curve \\
\hline WindTurbine $\beta$ VCurve & Curve & $\begin{array}{l}\text { GenerationD } \\
\text { ynamics }\end{array}$ & Wind Turbine $\beta$ V Curve \\
\hline WindTurbine $\omega$ VCurve & Curve & $\begin{array}{l}\text { GenerationD } \\
\text { ynamics }\end{array}$ & Wind Turbine $\omega \mathrm{V}$ Curve \\
\hline $\begin{array}{l}\text { WindTurbineDynamicsM } \\
\text { odel }\end{array}$ & & Domain & $\begin{array}{c}\text { Wind Turbine Dynamic } \\
\text { Model }\end{array}$ \\
\hline
\end{tabular}

\section{Conclusion}

Based on the provincial dispatch automation system's requirement of the dispatching of distributed wind plant, this paper extends wind plant CIM and wind turbine dynamic CIM, and meets the provincial grid's data and functionality requirements of the distributed wind plant.

\section{Acknowledgements}

In this paper, the research was sponsored by the Project of Science and Technology of State Grid Corp (Project No. 5442NY130007).

\section{References}

[1] Jian LUO, Botong ZhU, Ming CAI, et al. Interoperability of CIM and SCL model based on CIM XML [J]. Power System Protection and Control, 2011, (17): 134-138.

[2] Wei CUI, Yong ShI, Bing SUN. The construction and integration of grid model based on IEC61970/61968 [J]. Power System Protection Control, 2011, (17): 60-63.

[3] Hongbin SUN, Wenchuan WU, Boming ZHANG, et al. Application of extension of IEC61970 standard in control center integration [J]. Power System Technology, 2005, Vol. 29(6): 21-25.

[4] Wenchuan WU, Pengfei LIU, Yi ZHANG, et al. CIM extension of active distribution network and design of its standard example [J]. Power System Technology, 2012, Vol. 36(9): 155-161.

[5] Xiaoshu HUANG, Changguo ZHAI, Zhigang CAO, et al. A three-layer modeling method of new energy sources in power monitoring systems [J]. Automation of Electric Power System, 2012, Vol. 36(11): 72-76. 
[6] Yi LUO, Lin SHI, Guangyu TU, et al. Microgrid common information model applicable to plug and play feature of distributed resources [J]. Automation of Electric Power System, 2010, Vol. 34(8): 97-100.

[7] Haixuan LIU, Fubao WU, Daxing DONG, et al. Extended common information model for microgrid energy management system [J]. Automation of Electric Power System, 2012, Vol. 36(6): 45-51.

[8] Ming DING, Zhengkai ZHANG, Rui BI. Distributed Generation System Oriented CIM Extension [J]. Automation of Electric Power System, 2008, Vol. 32(20): 83-87.

[9] Yin DING, Ming DING, Rui BI, et al. Research on CIM/XML model of microgrid system [J]. Power System Protection and Control, 2010, Vol. 5(1): 37-42. 